\title{
Apelin-12 improves metabolic and functional recovery of rat heart after global ischemia
}

\author{
Oleg I. Pisarenko*, Valentin S. Shulzhenko, Yulia A. Pelogeykina, Irina M. Studneva, Denis N. \\ Khatri
}

Russian Cardiology Research-and-Production Complex, Moscow, Russia; *Corresponding Author: olpi@cardio.ru

Received 27 March 2010; revised 24 April 2010; accepted 26 April 2010.

\begin{abstract}
This work was designed to explore efficacy of apelin-12 (A-12) as a cardioprotective agent when given before ischemia or at reperfusion using the isolated working heart model. Hearts of male Wistar rats were subjected to $30-\mathrm{min}$ stabilization period followed by $35-\mathrm{min}$ global ischemia and 30-min reperfusion. A short-term infusion of Krebs-Henseleit buffer (KHB) containing $\mathrm{A}-12(35,70,140,280$ or $560 \mu \mathrm{M})$ was applied prior to ischemia (A-12-I) or at onset of reperfusion (A-12-R). KHB infusion was used as control. A-12 infusions induced a dose-dependent increase in recovery of coronary flow, contractile and pump function during reperfusion, with the largest augmentation of these indices in the A-12-I group. Both A-12 groups exhibited a significant reduction of LV diastolic pressure rise during reperfusion compared with control. Enhanced functional recovery in the A-12-I group was combined with a decrease in LDH leakage in perfusate on early reperfusion (by $36 \%$ vs. control, $p<0.05$ ). Preischemic infusion of $140 \mu \mathrm{M}$ A-12 markedly increased myocardial ATP content, enhanced preservation of the total adenine nucleotide pool and improved recovery of the energy charge in reperfused hearts. There was a trend towards increase in myocardial phosphocreatine by the end of reperfusion in the A-12-I group; however this benefit did not reach statistical significance. At the end of reperfusion, myocardial lactate and lactate/pyruvate ratio were on average 5-fold lower in A-12-I treated hearts compared with control ones and did not differ significantly from the initial values. Therefore, improved cardiac dysfunction after I/R injury and less cell membrane damage induced by A-12 are associated with maintaining high energy phosphates, particularly ATP, in reperfused myocardium. Changes
\end{abstract}

in energy metabolism may play a role in mechanisms of cardioprotection afforded by A-12 during I/R stress.

Keywords: Apelin-12, Rat Heart; Ischemia/ Reperfusion Injury; Energy Metabolism; Cell Membrane Damage

\section{INTRODUCTION}

Myocardial ischemia and subsequent reperfusion lead to formation of a number of intrinsic factors which mediate the cellular mechanisms of adaptation to altered oxygen and energy supply [1-3]. One of them is adipocytokine apelin, recently isolated from bovine stomach extracts and identified as the endogenous ligand of the human orphan G protein-coupled receptor APJ. Apelin is a 36amino acid peptide derived from 77-amino acid precursor proapelin, for which cDNAs have been cloned from humans, cattle, rats, and mice [4-6]. Apelin and its receptor are widely expressed in mammalian tissues; in the cardiovascular apelin/APJ system was found in the endothelial cells of small intramyocardial and pulmonary vessels, in coronary arteries, in endocardial endothelium cells, and in vascular smooth muscle cells [5,7]. Activation of apelin/APJ receptor system triggers cell-signaling mechanisms and induces positive inotropic and hypotensive effects in normal and failing myocardium [7-9]. These effects imply an important role of apelin in the regulation of cardiovascular homeostasis, making it an attractive target for heart failure therapy.

Only a few studies showed that at least two isoforms of apelin, apelin-13 and the physiologically less potent peptide, apelin-36, are capable to attenuate myocardial ischemia/reperfusion ( $\mathrm{I} / \mathrm{R})$ injury. Thus, administration of either of these peptides reduced infarct size and improved contractile function recovery of rat and mouse hearts after regional or global ischemia [10-12]. In cultured neonatal cardiomyocytes, apelin-13 decreased for- 
mation of reactive oxygen species (ROS) and malonic dialdehyde (MDA) with simultaneous augmentation of superoxide dismutase (SOD) activity and reduction of myocardial LDH leakage under hypoxia/reoxygenation treatment [12]. Some authors suggest that vasodilator and antioxidant features of apelin are related to an increase in NO formation due to promoted eNOS expression [13]. However, a failure of apelin-13 to influence eNOS phosphorylation was observed in mouse heart subjected to global ischemia and reperfusion [10]. Apelin-induced suppression of apoptosis, the delayed opening of the mitochondrial permeability transition pore (mPTP) and reduced cardiomyocyte contracture have been demonstrated in various experimental myocardial I/R models, including isolated cardiomyocytes, perfused hearts and hearts in situ $[10,12]$. One of the possible mechanisms whereby the apelin-APJ system may protect the myocardium form ischemia/reperfusion injury may be its actions on the reperfusion injury salvage kinase (RISK) pathway $[14,15]$. Abolishment of the protective effects of apelin by PI3K-Akt and ERK specific inhibitors suggests the activation of both kinase cascades by apelin [10]. These data, and especially inhibition of mPTP opening [16], indicate an implication of metabolic component in mechanisms of apelin action on ischemic heart.

To date, there is no information available regarding direct effects of the apelin/APJ-system on cardiac energy metabolism. Therefore, the objective of the present study was to evaluate alterations in metabolic state of postischemic heart occurring after apelin administration before ischemia or at onset of reperfusion. To test the potential metabolic importance of apelin, we used a 12 amino acid peptide, apelin-12, which is identical between human and rats and presumably has higher activity than longer C-terminal fragments [17].

\section{MATERIALS AND METHODS}

\subsection{Synthesis of Apelin-12}

A-12 (chemical structure H-Arg-Pro-Arg-Leu-Ser-HisLys-Gly-Pro-Met-Pro-Phe-OH, Mw 1422.7) was synthesized by the automatic solid phase method using an Applied BioSystems 431A peptide synthesizer (Germany) and Fmoc technology. The synthesized peptide was purified by preparative HPLC and identified by ${ }^{1} \mathrm{H}-\mathrm{NMR}$ spectroscopy and mass spectrometry.

\subsection{Heart Perfusion}

The present investigation conforms to the Guide for Care and Use of Laboratory Animals published by the US National Institute of Health (NIH publication No. 85-23; revised 1985). Male Wistar rats (280-300 g) were heparinized (500 U i.p.) and anaesthetized with urethane (1.3 g/kg body weight). Hearts (1.4-1.5 g wet wt) were excised and immediately placed into ice-cold KrebsHenseleit bicarbonate buffer (KHB) until contraction stopped. The aorta was then cannulated and Langendorff perfusion was performed at a constant pressure equivalent to $75 \mathrm{~cm} \mathrm{H}_{2} \mathrm{O}$ for $15 \mathrm{~min}$. Working perfusion was performed according to a modified method of Neely under constant left atrium pressure and aortic pressure (AP) of 20 and $100 \mathrm{~cm} \mathrm{H}_{2} \mathrm{O}$, respectively. $\mathrm{KHB}$ containing (in $\mathrm{mM}$ ): $\mathrm{NaCl} 118 ; \mathrm{KCl} 4.7 ; \mathrm{CaCl}_{2} 3.0 ; \mathrm{Na}_{2}$ EDTA 0.5; $\mathrm{KH}_{2} \mathrm{PO}_{4}$ 1.2; $\mathrm{MgSO}_{4}$ 1.2; $\mathrm{NaHCO}_{3}$ 25.0; glucose 11.0. was oxygenated with a mixture of $95 \% \mathrm{O}_{2}$ and $5 \% \mathrm{CO}_{2}$; $\mathrm{pH}$ was $7.4 \pm 0.1$ at $37^{\circ} \mathrm{C}$; it was passed through a $5 \mu \mathrm{m}$ Millipore filter (Bedford, MA, USA) before use. A needle was inserted into the left ventricular cavity to registered LV pressure via a Gould Statham P50 transducer, SP 1405 monitor and a Gould Brush SP 2010 recorder (Gould, Oxnard, Ca, USA). The contractile function index was calculated as the LV developed pressure-heart rate product (LVDPxHR), where LVDP is the difference between LV systolic and LV end-diastolic pressure. Cardiac pump function was assessed by minute volume and aortic output; stroke was calculated as cardiac output/ HR. Coronary resistance was calculated as AP/coronary flow [18].

\subsection{Experimental Protocol}

After preliminary Langendorff perfusion, the hearts were perfused in working mode for $20 \mathrm{~min}$; the steady state values of cardiac function were recorded at the end of this period. Then, the hearts were randomly assigned onto one of three groups: 1) control $(\mathrm{n}=13)$; 2) A-12 treated before global ischemia (A-12-I, $\mathrm{n}=13)$; 3) A-12 treated at onset of reperfusion $(A-12-R, n=13)$. After the steady state period, the control hearts were perfused in Langendorff mode for $5 \mathrm{~min}$ at a constant flow rate of $4 \mathrm{ml} / \mathrm{min}$ and then they were subjected to 35-min normothermic global ischemia followed by 5-min Langendorff perfusion with subsequent 30-min working reperfusion. In the A-12-I group, 5-min Langendorff perfusion at a constant flow rate of $4 \mathrm{ml} / \mathrm{min}$ with $\mathrm{KHB}$ containing A-12 $(35,70,140,280$ or $560 \mu \mathrm{M})$ was applied prior to global ischemia. The hearts of the A-12-R group were perfused with KHB containing A-12 in the same mode after global ischemia. After preliminary working perfusion (steady state) and at the end of reperfusion, the hearts were freeze-clamped in liquid nitrogen for metabolite analysis. The myocardial effluent was collected in ice-cold containers during both periods of Langendorff perfusion for immediate measurement of $\mathrm{LDH}$ activity. The total number of hearts used for functional and metabolic determinations was 49 . 


\subsection{Tissue Sampling, Metabolite Analysis and Assay of LDH Activity.}

Frozen tissue was quickly homogenized in cooled $6 \%$ perchloric acid $(10 \mathrm{ml} / \mathrm{g})$ using an Ultra-Turrax T-25 homogenizer (IKA-Labortechnik, Staufen, Germany), and the homogenates were centrifuged at $2500 \mathrm{~g}$ for $10 \mathrm{~min}$ at $4^{\circ} \mathrm{C}$. The supernatants were then neutralized with $5 \mathrm{M}$ $\mathrm{K}_{2} \mathrm{CO}_{3}$ to $\mathrm{pH} 7.4$ and the extracts were centrifuged after cooling to remove $\mathrm{KClO}_{4}$ precipitate. Tissue dry weights were determined by weighing a portion of the pellets after extraction with perchloric acid and drying overnight at $110^{\circ} \mathrm{C}$. Concentrations of ATP, ADP, AMP, phosphocreatine and lactate in neutralized tissue extracts were determined specrtophotometrically by enzymatic methods [19-22]. A modified UV-spectroscopy method was used to assay tissue extracts for pyruvate [23]. Determination of metabolite in each tissue extract was performed three times to calculate the average metabolite concentration in a sample. $\mathrm{LDH}$ activity in the myocardial effluent was measured according to the method of Bergmeyer and Bernt [24] using pyruvate as substrate. Determination of LDH activity in the perfusate was repeated twice in each sample. Enzymes and chemicals were purchased from Sigma Chemical Co. (St Louis, MO USA). Solutions were prepared using deionized water (Milli Ro-4; Milli-Q, Millipore Corp. Bedford, MA, USA).

\subsection{Statistical Analysis}

Statistical differences between more than two groups were evaluated by one-way analysis of variance (ANOVA) and followed by Scheffe F-test. Comparisons between two groups involved use of the Student's t test. A $\mathrm{p}<0.05$ was considered statistically significant.

\section{RESULTS}

\subsection{Postischemic Recovery of Cardiac Function}

Administration of A-12 before global ischemia or at onset of reperfusion enhanced recovery of cardiac pump function during reperfusion. As an example, a dosedependent increase in recovery of cardiac output (CO) by the end of reperfusion is shown on Figure 1(a). The significant increase in $\mathrm{CO}$ recovery was observed after pre- or postischemic infusion of $70 \mu \mathrm{M} \mathrm{A}-12$ as compared with the control. The differences between $\mathrm{CO}$ recovery in the experimental groups and control became more pronounced with an increase in A-12 concentration in KHB. The maximal response to A-12 was observed at the concentration of $280 \mu \mathrm{M}$; at higher concentrations a dose-effect curve reached a plateau. Within the range of $70-560 \mu \mathrm{M}$, the peptide administration prior to ischemia was more effective than after it. Thus, the maximal restoration of $\mathrm{CO}$ after infusion $560 \mu \mathrm{M}$ A-12 were $86 \pm$ $9 \%$ and $59 \pm 6 \%$ of the steady state value in the group A-12-I and A-12-R, correspondingly, in comparison with $32 \pm 2 \%$ in the control $(\mathrm{P}<0.02-0.01)$. A similar dosedependent effects were obtained for recovery of the LVDPXHR product during reperfusion in both experimental groups (Figure 1(b)).

Recovery of cardiac and coronary function indices at the end of reperfusion after administration of $140 \mu \mathrm{M} \mathrm{A}$ -12 before and after of ischemia is presented in Table 1. In addition to $\mathrm{CO}$, aortic output and stroke volume were also significantly higher in the A-12-I group than in the A-12-R group and in control. The especially greater differences between the groups were noted for aortic output: in the A-12-I group its recovery was twice more effective than in the A-12-R group and 30 times higher than in control. An enhanced recovery of cardiac pump function in A-12 treated hearts was accompanied by an augmented restoration of the LVDPxHR product. This effect was due to higher recovery of HR and in both A-12 groups comparing with control. A significant increase in the values of LVDP was in turn caused by a marked reduction of LV diastolic pressure during reperfusion (Figure 2). Beneficial effects of A-12 on cardiac function were accompanied by the peptide influence on the coronary system. Thus, both of A-12 groups exhibited a significant increase in coronary flow with concomitant reduction in coronary resistance in comparison with control (Table 1) indicating a participation of coronary vessels in response to A-12 administration.

\subsection{The Energy State of Reperfused Hearts}

Changes in the myocardial content of adenine nucleotides and the end products of anaerobic glycolysis, lactate and pyruvate, at the end of reperfusion caused by preischemic infusion of $140 \mu \mathrm{M} \mathrm{A}-12$ are compared with the initial myocardial levels of these metabolites in Table 2. By the end of reperfusion, the control group showed a profound decrease in the ATP content (to $37 \pm$ $7 \%$ of the initial value) with a simultaneous increase in ADP and AMP levels (on the average 1.7 and 5.6 times, respectively). These changes indicated the preferred degradation of adenine nucleotides, whose total myocardial pool $(\Sigma \mathrm{AN}=\mathrm{ATP}+\mathrm{ADP}+\mathrm{AMP})$ was lowered to $58 \pm 3 \%$ of the steady state value. The lactate and pyruvate contents were, correspondingly, 5- and 1.3-fold higher than the normal values, thus indicating an inhibition of glucose oxidation during reperfusion. Administration of $140 \mu \mathrm{M} \mathrm{A-12}$ before ischemia enhanced ATP preservation by $60 \pm 3 \%$ and twice decreased AMP content in the reperfused hearts. As a result the myocardial $\Sigma$ AN pool was preserved considerably better than in the 
control and composed $81 \pm 6 \%$ of the steady state value. Redistribution in the adenine nucleotide content considerably increased the total energy charge $(\mathrm{EC}=(\mathrm{ATP}+$ $0.5 \mathrm{ADP}) / \mathrm{ATP}+\mathrm{ADP}+\mathrm{AMP}$ ) in the A-12-I group as compared with the control. The observed improvement in the energy state of reperfused hearts was combined with a decrease in myocardial lactate content to the steady state value (Table 2). Although the myocardial pyruvate level in the A-12-I group remained raised at the end of reperfusion, the lactate/pyruvate ratio was 5 times lower than in the control and did not differ significantly from the steady state value.

Preischemic administration of $140 \mu \mathrm{M}$ A-12 did not significantly enhance recovery of phosphocreatine (PCr): by the end of reperfusion its myocardial content was $14.95 \pm 1.32 \mu \mathrm{mol} / \mathrm{g}$ of dry wt vs. $12.02 \pm 2.61 \mu \mathrm{mol} / \mathrm{g}$ of dry wt in the control. The total creatine pool $(\Sigma \mathrm{Cr}=$ $\mathrm{PCr}+$ creatine) in A-12-treated hearts did not differ sig-

Table 1. Effects of apelin-12 administration before or after global ischemia on functional recovery of isolated rat at the end of reperfusion.

\begin{tabular}{|c|c|c|c|c|}
\hline & $\begin{array}{l}\text { Steady state } \\
(\mathrm{n}=39)\end{array}$ & $\begin{array}{l}\text { Control } \\
(\mathrm{n}=13)\end{array}$ & $\begin{array}{l}\text { A-12-R } \\
(\mathrm{n}=13)\end{array}$ & $\begin{array}{l}\text { A-12-I } \\
(\mathrm{n}=13)\end{array}$ \\
\hline $\begin{array}{l}\text { Coronary flow, } \\
\mathrm{ml} / \mathrm{min}\end{array}$ & $19.1 \pm 2.0$ & $14.5 \pm 0.4$ & $15.9 \pm 1.1$ & $17.8 \pm 0.9^{\mathrm{a}}$ \\
\hline $\begin{array}{l}\text { Perfusion pressure, } \\
\mathrm{mm} \mathrm{Hg}\end{array}$ & $64 \pm 6$ & $60 \pm 1$ & $61 \pm 1$ & $63 \pm 1^{\text {a }}$ \\
\hline $\begin{array}{l}\text { Coronary resistance, } \\
\mathrm{mm} \mathrm{Hg} / \mathrm{ml}\end{array}$ & $3.46 \pm 0.04$ & $4.19 \pm 0.07$ & $3.98 \pm 0.21$ & $3.78 \pm 0.17^{\mathrm{a}}$ \\
\hline $\begin{array}{l}\text { LV systolic pressure, } \\
\mathrm{mm} \mathrm{Hg}\end{array}$ & $102 \pm 1$ & $70 \pm 1$ & $80 \pm 3^{a}$ & $92 \pm 3^{a b}$ \\
\hline $\begin{array}{l}\mathrm{LV} \text { diastolic pressure, } \\
\mathrm{mm} \mathrm{Hg}\end{array}$ & $-2 \pm 1$ & $8 \pm 1$ & $5 \pm 1^{a}$ & $1 \pm 1^{\mathrm{ab}}$ \\
\hline $\begin{array}{l}\text { LV developed pressure, } \\
\mathrm{mm} \mathrm{Hg}\end{array}$ & $106 \pm 1$ & $62 \pm 1$ & $75 \pm 4^{\mathrm{a}}$ & $92 \pm 4^{\mathrm{ab}}$ \\
\hline $\begin{array}{l}\text { Heart rate, } \\
\text { beat } / \mathrm{min}\end{array}$ & $310 \pm 2$ & $242 \pm 3$ & $264 \pm 6^{a}$ & $288 \pm 6^{a b}$ \\
\hline $\begin{array}{l}\text { LVDP x HR, } \\
\mathrm{mm} \mathrm{Hg} / \mathrm{min}\end{array}$ & $33062 \pm 624$ & $14877 \pm 561$ & $20167 \pm 1653^{a}$ & $26780 \pm 1653^{a b}$ \\
\hline $\begin{array}{l}\text { Aortic output, } \\
\mathrm{ml} / \mathrm{min}\end{array}$ & $28.1 \pm 3.0$ & $0.6 \pm 0.3$ & $8.7 \pm 0.8^{a}$ & $19.1 \pm 1.3^{\mathrm{ab}}$ \\
\hline $\begin{array}{l}\text { Cardiac output, } \\
\mathrm{ml}\end{array}$ & $46.2 \pm 1.1$ & $14.8 \pm 0.9$ & $24.0 \pm 2.3^{\mathrm{a}}$ & $35.5 \pm 2.2^{\mathrm{ab}}$ \\
\hline Stroke volume, $\mu \mathrm{l}$ & $149 \pm 1$ & $61+3$ & $91+7^{\mathrm{a}}$ & $127+4^{a b}$ \\
\hline
\end{tabular}

The hearts were perfused as indicated in Materials and methods. Steady state, 20-min preliminary working perfusion; Control, 5-min Langendorff perfusion +35 -min global ischemia +5 -min Langendorff perfusion +30 -min working reperfusion; A-12-R, 5-min Langendorff perfusion with 140 $\mu \mathrm{M}$ A-12 after global ischemia; A-12-I, 5-min Langendorff perfusion with $140 \mu \mathrm{M}$ A-12 before global ischemia. Data are the mean \pm SEM. ${ }^{a} \mathrm{p}<0.05$ vs. control group; ${ }^{\mathrm{b}} \mathrm{p}<0.05$ vs. A-12-R group.

Table 2 Effects of $140 \mu \mathrm{M}$ apelin-12 infusion before global ischemia on myocardial content of metabolites and indices of myocardial energy state at the end of reperfusion.

\begin{tabular}{|c|c|c|c|}
\hline & Steady state & Control & A-12-I \\
\hline ATP & $22.42 \pm 2.06$ & $8.30 \pm 1.73^{\mathrm{a}}$ & $13.11 \pm 0.69^{\mathrm{ab}}$ \\
\hline $\mathrm{ADP}$ & $2.80 \pm 0.12$ & $4.86 \pm 0.28^{\mathrm{a}}$ & $5.90 \pm 0.28^{\mathrm{a}}$ \\
\hline AMP & $0.71 \pm 0.01$ & $3.97 \pm 0.74^{\mathrm{a}}$ & $1.92 \pm 0.06^{\mathrm{ab}}$ \\
\hline$\Sigma \mathrm{AN}$ & $25.93 \pm 1.45$ & $17.13 \pm 1.42^{\mathrm{a}}$ & $20.94 \pm 0.92^{\mathrm{ab}}$ \\
\hline ATP/ADP & $8.00+0.10$ & $1.72 \pm 0.35^{\mathrm{a}}$ & $2.23 \pm 0.07^{\mathrm{a}}$ \\
\hline $\mathrm{EC}$ & $0.91 \pm 0.02$ & $0.60 \pm 0.06^{\mathrm{a}}$ & $0.77 \pm 0.01^{\mathrm{ab}}$ \\
\hline Lactate & $1.72 \pm 0.19$ & $10.48 \pm 3.08^{\mathrm{a}}$ & $2.12 \pm 0.48^{b}$ \\
\hline Pyruvate & $0.18 \pm 0.02$ & $0.23 \pm 0.03$ & $0.22 \pm 0.02$ \\
\hline Lactate/Pyruvate & $9.55 \pm 0.98$ & $45.56 \pm 7.34^{\mathrm{a}}$ & $9.64 \pm 1.25^{b}$ \\
\hline
\end{tabular}

Steady state, 20-min preliminary working perfusion; Control, 5-min Langendorff perfusion +35 -min global ischemia +5 -min Langendorff perfusion + 30-min working reperfusion; A-12-I, 5-min Langendorff perfusion with $140 \mu \mathrm{M} \mathrm{A-12} \mathrm{before} \mathrm{global} \mathrm{ischemia.} \mathrm{Data} \mathrm{are} \mathrm{the} \mathrm{mean} \pm$ SEM for 10 experiments. Metabolite contents are expressed in $\left(\mu \mathrm{mol} / \mathrm{g}\right.$ dry wt). ${ }^{\mathrm{a}} \mathrm{p}<0.05$ vs. steady state; ${ }^{\mathrm{b}} \mathrm{p}<0.05 \mathrm{vs}$. control. $\Sigma \mathrm{AN}=\mathrm{ATP}+\mathrm{ADP}+\mathrm{AMP}$. The energy charge $(\mathrm{EC})=(\mathrm{ATP}+0.5 \mathrm{ADP}) / \mathrm{ATP}+\mathrm{ADP}+\mathrm{AMP}$. 
nificantly from this index in the control and in the steady state being $59.65 \pm 156,56.65 \pm 3.89$ and $59.26 \pm 1.87$ $\mu \mathrm{mol} / \mathrm{g}$ of dry $\mathrm{wt}$, correspondently.

\subsection{Lactate Dehydrogenase Leakage}

The ability of A-12 to influence cell membrane damage was assessed by changes in $\mathrm{LDH}$ release into the myocardial effluent before and after global ischemia (Table 3). For the 5-min period of $140 \mu \mathrm{M} \mathrm{A}-12$ infusion prior to ischemia, LDH leakage did not differ significantly from that one in control. Therefore A-12 administration did cause damage to the sarcolemma of nonischemic cardiomyocytes. During the 5-min period after ischemia, the release of LDH activity in the perfusate of the control group was increased on average 2.6 times compared with this value before ischemia indicating $\mathrm{I} / \mathrm{R}$ membrane damage. However in the A-12-I group, the postischemic LDH leakage was reduced by $40 \%$ compared with control. This finding suggested fewer membrane defects determining a release of cytoplasmic LDH from the myocardium.

\section{DISCUSSION}

The present study demonstrates, for the first time, cardioprotective properties of exogenous A-12 in isolated working rat heart subjected to global ischemia and reperfusion. They are manifested by enhanced contractile and pump function recovery and a better restoration of coronary flow, which are more effective after the peptide administration before ischemia than at onset of reperfusion. (Figure 1, Table 1). The obtained data are in broad agreement with the previous observations demonstrating a reduction of ischemia-reperfusion injury induced by exogenous apelin-13, Pyr $^{1}$-apelin-13 and apelin-36 in Langendorff perfused mouse and rat hearts [10-12,25]. It is essential that a higher functional level of postischemic hearts protected by A-12 in our experiences is combined with augmented metabolic state. Probably this beneficial effect is associated with enhanced oxidation of glucose, the main energy substrate of isolated perfused hearts. Indeed, A-12 treated hearts exhibit substantially reduced lactate/pyruvate ratio as compared with control and the normal myocardial lactate content at the end of reperfusion. In parallel, A-12 administration exerts an energy conservation effect evidenced by higher myocardial ATP and $\Sigma$ AN contents and enhanced EC of postischemic cardiomyocytes. In addition, augmentation of energy state of postischemic hearts by pretreatment with 140 $\mu \mathrm{M}$ A-12 is accompanied by less cell membrane damage on early reperfusion (Table 3). Taken together, these findings indicate that alterations in myocardial metabolism induced by apelin may play a role in improving cardiac dysfunction after ischemia and reperfusion stress.

Table 3. Effects of $140 \mu \mathrm{M}$ apelin-12 infusion on $\mathrm{LDH}$ leakage before and after global ischemia.

\begin{tabular}{lcc}
\hline & Before ischemia & After ischemia \\
\hline Control $(n=6)$ & $28.60 \pm 3.01$ & $73.64 \pm 5.06^{\sigma}$ \\
A-12-I $(n=7)$ & $24.28 \pm 2.73$ & $53.56 \pm 6.52^{\text {a b }}$ \\
\hline
\end{tabular}

Control, 5-min Langendorff perfusion +35 -min global ischemia + 5-min Langendorff perfusion +30 -min working reperfusion; A-12-I, 5 -min infusion of $140 \mu \mathrm{M} \mathrm{A}-12$ before global ischemia. Values are the means \pm SEM and are expressed in IU/g dry wt. for 5-min Langendorff perfusion before or after global ischemia. ${ }^{a} \mathrm{p}<0.05$ vs. control group; ${ }^{\mathrm{b}} \mathrm{p}<0.05$ vs. the value before ischemia.
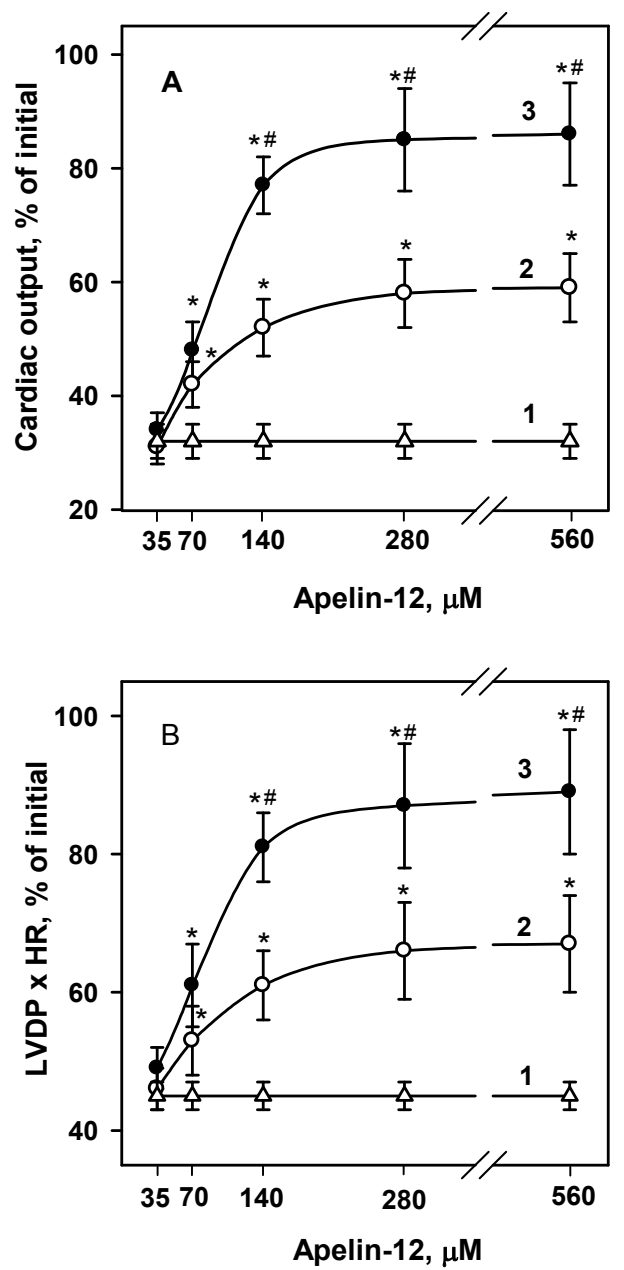

Figure 1. Effects of A-12 concentration in KHB on recovery of minute volume (A) and the LVDP-HR product (B) at the end of reperfusion. 1) 5-min Langendorff perfusion + 35-min global ischemia +5 -min Langendorff perfusion +30 -min working reperfusion (control); 2) 5-min Langendorff perfusion with A-12 after global ischemia (A-12-R); 3) 5-min Langendorff perfusion with A-12 before global ischemia $(\mathrm{A}-12-\mathrm{I})$. Data are the mean \pm SEM for $12-15$ experiments. ${ }^{*} \mathrm{p}<0.05$ vs. control; ${ }^{\#} \mathrm{p}<0.05$ vs. A-12-R. 


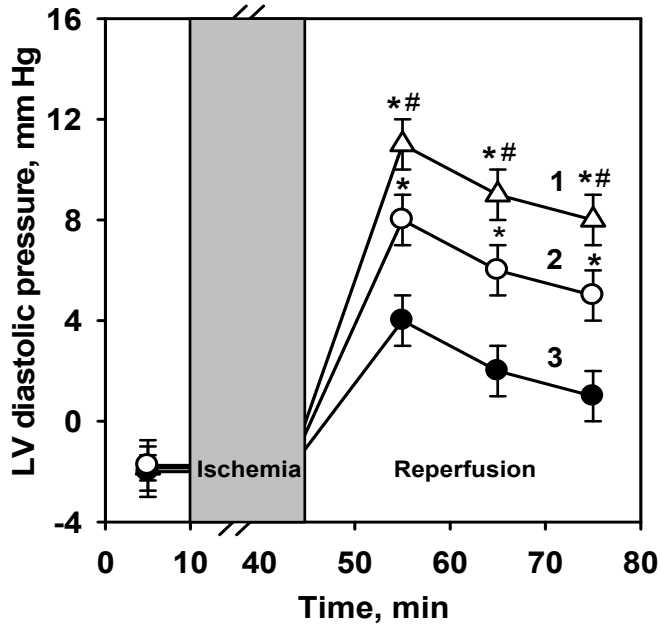

Figure 2. Effects of A-12 administration before or after global ischemia on recovery of LV diastolic pressure during reperfusion. 1) 5-min Langendorff perfusion +35 -min global ischemia +5 -min Langendorff perfusion +30 -min working reperfusion (control); 2) 5-min Langendorff perfusion with $140 \mu \mathrm{M}$ A-12 after global ischemia (A-12-R); 3) 5-min Langendorff perfusion with $140 \mu \mathrm{M}$ A-12 before global ischemia (A-12-I). Data are the mean \pm SEM for $12-15$ experiments. ${ }^{*} \mathrm{p}<0.05$ vs. control; ${ }^{\#} \mathrm{p}<0.05$ vs. A-12-R.

The current studies evaluating the mechanisms whereby apelin confer protection against $\mathrm{I} / \mathrm{R}$ injury have yielded mixed results. Wide discrepancy relates to variations in apelin/APJ expression. In ventricular cardiomyocytes isolated from rats, apelin expression is increased under hypoxia presumably via hypoxia-inducible factor-mediated pathway $[12,26]$. Apelin and APJ are upregulated in the heart and skeletal muscle following myocardial injury in the murine model of LAD occlusion in vivo [27]. Accordingly, endogenous myocardial apelin and APJ expression are increased in failing rat hearts in compensation for ischemic cardiomyopathy [28]. In contrast, apelin content in plasma, atrial and ventricular myocardium and APJ gene expression decrease in myocardial injury induced by repeated isoproterenol injections in rats [29]. The endogenous apelin/ APJ system is compensatory up-regulated and ultimately down-regulated during sustained myocardial ischemia in vitro which was mimicked by glucose deprivation [30]. In isolated rat heart, apelin and APJ mRNA are upregulated during ischemia but return to the control levels following reperfusion [11]. Based on these conflicting data we suggest that in acute ischemic myocardial injury, the endogenous apelin/APJ system may have a protective role. However the endogenous apelin production is insufficient for activation of the APJ receptors to reduce myocardial ischemia-reperfusion injury. This assumption is consistent with cardioprotective activity of exogenous isoforms of apelin revealed in different experimental models [7,10-12,28-30].

Effects of exogenous apelin are mediated partly by activation of components of the reperfusion injury salvage kinase (RISK) pathway, phosphatidylinositol-3-OH kinase (PI3K)/Akt, p44/42 mitogen-activated protein kinase (MAPK) extracellular signal-regulated MAPK (ERK1/2) $[10,14,16,31]$. This is confirmed by that fact that the inhibitors of PI3K-Akt and p44/42 phosphorylation, LY294002 and UO126, respectively, abolished reduction of infarct size induced by apelin-13 in vitro [10]. Similarly, addition of wortmannin and PD098059, the inhibitors of PI3K/Akt and ERK1/2, to culture medium during hypoxia/reoxygenation, suppressed cardiomyocyte viability provided by apelin-13 [12]. Further evidence that apelin may act via activation of the RISK pathway was obtained from the findings concerning the MPTP opening, which causes the energy collapse and irreversible cardiomyocyte damage [16,32]. Thus, while apelin-13 delayed the times until mitochondrial membrane depolarization and rigor contracture, LY294002 and mitogenactivated protein kinase (MEK) inhibitor 1 blocked these effects [10]. Anti-apoptotic effects of apelin-13 in glucose-deprived cardiomyocytes involve activation of the PI3K/Akt and the mammalian target of rapamycin (mTOR) pathways [30]. As follows from the study by Kleinz and Baxter [11], the RISK cascades are not always used for myocardial protection afforded by apelin. These authors showed that apelin-13 administered during reperfusion significantly reduced infarct size in rat hearts subjected to coronary occlusion followed by reperfusion. However this protective effect was not abolished by co-administration of the PI3K/Akt inhibitor wortmannin or the P70S6 kinase inhibitor rapamycin. Therefore, apelin may exhibit cardioprotection using alternative mediators and signaling pathways.

Elevation of eNOS expression induced by apelin [8, 12] may lead to increase in NO formation, contributing to recovery of cardiac function and metabolism after ischemia. In our study, it is indirectly confirmed by significant improvement of coronary flow in the A-12-I group during reperfusion (Table 1). Involvement of the L-Arg/NOS/NO pathway in vascular function regulation by apelin was noted earlier Tatemoto et al. [4]. They demonstrated that in anaesthetized rats the hypotensive effect of apelin-12 (to a greater extent than apelin-13 and apelin-36) is accompanied by an increase in the total nitrite and nitrate in plasma, and is abrogated in the presence of N-nitro-L-arginine methyl ester (L-NAME), a NOS inhibitor. Subsequently, a dose-dependent reduction of the mean arterial pressure during apelin infusion was documented in conscious rats [33]. It is equally important that during myocardial ischemia and reperfusion, NO generated by vascular and endocardial endothelial NOS may attenuate oxidative stress by scavenging reactive oxygen species (ROS) and reduce cell death via 
inhibition of mPTP $[34,35]$. The role of NO, formed under apelin administration, in reducing I/R injury so far remains unclear. However, the antioxidant properties of exogenous apelin-13 were confirmed by parallel decrease in ROS and MDA formation in the isolated rat heart and cardiomyocyte culture during simulation of ischemic and reperfusion stress [12]. It is possible that these effects were due not only to eNOS expression, but also to apelin-stimulated increase in SOD activity, which is inhibited in myocardial tissue by $\mathrm{I} / \mathrm{R}[12,36]$.

In addition to the above mechanisms the results of our study clearly indicate that enhanced energy state of reperfused myocardium is implicated in amelioration of I/R injury by apelin. In fact, a better preservation of myocardial adenine nucleotides, and especially ATP, may be of critical importance for maintaining cell membrane integrity and ion homeostasis, preventing contracture and ROS generation during reperfusion [37,38]. Presumably a more effective postischemic restoration of energy metabolism was related to promoted glucose oxidation in A-12 treated hearts (Table 2). Various studies point out an emerging role of apelin in glucose metabolism [39]. In particular, it was shown that apelin stimulates glucose utilization in normal and insulin- resistant mice [40]. In the heart and skeletal muscle, apelin effects on glucose uptake have been suggested to be associated with the activation of eNOS, AMP-activated protein kinase (AMPK) and Akt-dependent pathways $[41,43]$. Therefore apelin-stimulated alterations in glucose metabolism may represent a promising approach for correcting metabolic disorders induced by ischemia and reperfusion.

In conclusion, the present study revealed the ability of exogenous apelin-12 to improve cardiac dysfunction, sarcolemma integrity and myocardial metabolic state after I/R injury. Although apelin isoforms with shorter Cterminal fragments than apelin-36 have higher bioactivity, they may be subjected to enzymatic degradation in vivo with formation of inactive forms. Synthesis of modified analogues of apelin is able to increase their resistance to the action of aminopeptidases and lead to the development of pharmacological agonists for APJ receptor. This intriguing possibility may provide new therapeutic tools for treatment acute coronary syndrome and heart failure.

\section{ACKNOWLEDGEMENTS}

The authors thank Dr. Zh.D. Bespalova for synthesis of apelin-12 and discussion of the results.

\section{REFERENCES}

[1] Verma, S., Fedak, P.W., Weisel, R. D., Butany, J., Rao, V. and Maitland, A. (2002) Fundamentals of reperfusion injury for the clinical cardiologist. Circulation, 105(20), 2332-2336.

[2] Infanger, M., Faramarzi, S., Grosst, J., Kurth, E., Ulbrich, C. and Bauer J. (2007) Expression of vascular endothelial growth factor and receptor tyrosine kinases in cardiac ischemia/reperfusion injury. Cardiovascular Pathology, 16(5), 291-299.

[3] Smith. C.C., Mocanu, M.M., Davidson, S. M., Wynne, A. M., Simpkin, J. C. and Yellon D. M. (2006) Leptin, the obesity-associated hormone, exhibits direct cardioprotective effects. British Journal of Pharmacology, 149(1), 5-13.

[4] Tatemoto, K., Hosoya, M., Habata, Y., Fujii, R., Kakegawa, T., Zou, M. X., Kawamata, Y., Fukusumi, S., Hinuma, S., Kitada, C., Kurokawa, T., Onda, H. and Fujino, M. (1998) Isolation and characterization of a novel endogenous peptide ligand for the human APJ receptor. Biochemical and Biophysical Research Communications, 251(2), 471-476.

[5] Boucher, J., Masri, B., Daviaud, D., Gesta S., Guigne, C. and Mazzucotelli, A. (2005) Apelin, a newly identified adipokine up-regulated by insulin and obesity. Endocrinology, 146, 1764-1771.

[6] Lee, D. K., Cheng, R., Nguyen, T., Fan, T., Kariyawasam, A. P., Liu, Y., Osmond, D. H., George, S. R. and O'Dowd, B.F. (2000) Characterization of apelin, the ligand for the APJ receptor. Journal of Neurochemistry, 74(1), 34-41.

[7] Berry, M.F., Pirolli, T.J., Jayasankar, V., Burdick, J., Morine, K.J. and Gardner, T.J. (2004) Apelin has in vivo inotropic effects on normal and failing hearts. Circulation, 110(Supplement II), II187-II193.

[8] Tatemoto, K., Takayama, K., Zou, M.X., Kumaki, I., Zhang, W. and Kumano, K. (2001) The novel peptide apelin lowers blood pressure via a nitric oxide-dependent mechanism. Regulatory Peptides, 99(2-3), 87-92.

[9] Chen, M.M., Ashley, E.A., Deng, D.X., Tsalenko, A., Deng, A. and Tabibiazar, R. (2003) Novel role for the potent endogenous inotrope apelin in human cardiac dysfunction. Circulation, 108(12), 1432-1439.

[10] Simpkin, J. C., Yellon, D. M., Davidson, S.M., Lim, S. Y., Wynne, A. M. and Smith, C. C. (2007) Apelin-13 and apelin-36 exhibit direct cardioprotective activity against ischemia-reperfusion injury. Basic Research in Cardiology, 102(6), 518-528.

[11] Kleinz, M.J. and Baxter, G.F. (2008) Apelin reduces myocardial reperfusion injury independently of PI3K/Akt and P70S6 kinase. Regulatory Peptides, 146(1-3), 271277.

[12] Zeng, X. J., Zhang, L. K., Wang, H. X., Lu, L. Q., Ma, L. Q. and Tang, C.S. (2009) Apelin protects heart against ischemia/reperfusion injury in rat. Peptides, 30(6), 11441152.

[13] Jia, Y. X., Lu, Z.F., Zhang, J., Pan, C. S., Yang, J.H. and Zhao, J. (2007) Apelin activates L-arginine/nitric oxide synthase/nitric oxide pathway in rat aortas. Peptides, 28(10), 2023-2029.

[14] Masri, B., Lahlou, H., Mazarguil, H., Knibiehler, B. and Audigier, Y. (2002) Apelin (65-77) activates extracellular signal-regulated kinases via a PTX-sensitive G protein. Biochemical and Biophysical Research Communications, 290(1), 539-545. 
[15] Masri, B., Knibiehler, B. and Audigier, Y. (2005) Apelin signaling: A promising pathway from cloning to pharmacology. Cell Signaling, 17(4), 415-426.

[16] Tsang, A., Hausenloy, D.J., Mocanu, M.M. and Yellon, D.M. (2004) Postconditioning: A form of "modified reperfusion" protects the myocardium by activating the phosphatidylinositol 3-kinase-Akt pathway. Circulation Research, 95(3), 230-232.

[17] Kawamata, Y., Habata, Y. and Fukusumi, S. (2001) Molecular properties of apelin: tissue distribution and receptor binding. Biochimica et Biophysica Acta, 1538(2-3), 162-171.

[18] Pisarenko, O. I., Shulzhenko, V. S. and Studneva, I.M. (1999) Metabolic effects of carbachol and ischaemic preconditioning in isolated rat heart. Clinical and Experimental Pharmacology and Physiology, 26(1), 26-31.

[19] Lamprecht, W. and Trautschold, I. (1974) Creatine phosphate. Determination with CK, HK and G6P-DH. In: Bergmeyer, H. U., Ed., Methods of Enzymatic Analysis. Academic Press, New York, 1777-1781.

[20] Jaworek, D., Gruber, W. and Bergmeyer, H.U. (1974) Adenosine-5'-diphosphate and adenosine-5'-monophosphate. In: Bergmeyer, H. U., Ed., Methods of Enzymatic Analysis. Academic Press, New York, 2127-2131.

[21] Bernt, E., Bergmeyer, H. U. and Mollering H. (1974) Creatine. In: Bergmeyer, H.U., Ed., Methods of Enzymatic Analysis. Academic Press, New York, 1772-1776.

[22] Gutman, I., Wahlenfeld, A.W.L. (1974) L-(+)-Lactate. Determination with LDH and NAD. In: Bergmeyer, H.U., Ed., Methods of Enzymatic Analysis. Academic Press, New York, 1464-1467.

[23] Bucher, T., Czok, R., Lamprecht, W. and Latzko E. (1963) Pyruvate. In: Bergmeyer, H.U., Ed., Methods of enzymatic analysis. Academic Press, New York, 2253-2259.

[24] Bergmeyer, H.U. and Bernt, E. (1974) Lactate dehydrogenase. UV-assay with pyruvate and NADH. In: Bergmeyer, H.U., Ed., Methods of enzymatic analysis. Academic Press, New York, 574-578.

[25] Zeng, X.J., Wang, H.X., Lu, L.Q., Hao, G., Wang, X.Y. and Ma, L.Q. (2007) Effects and mechanisms of apelin-13 on ischemia/reperfusion injury in rat heart. Chinical Pharmacology Bull, 23(1), 82-85.

[26] Ronkainen, V.P., Ronkainen, J.J. and Hanninen, S.L. (2007) Hypoxia inducible factor regulates the cardiac expression and secretion of apelin. Journal of the Federation of American Societies for Experimental Biology, 21(8), 1821-1830.

[27] Sheikh, A.Y., Chun, H.J. and Glassford, A.J. (2008) In vivo genetic profiling and cellular localization of apelin reveals a hypoxia-sensitive, endothelial centered pathway activated in ischemic heart failure. American Journal of Physiological Heart and Circulatory Physiology, 294(1), H88-H98.

[28] Atluri, P., Morine, K.J. and Liao, G.P. (2007) Ischemic heart failure enhances endogenous myocardial apelin and A.P.J. receptor expression. Cellular and Molecular Biology Letter, 12(1), 127-138.

[29] Jia, Y.X., Pan, C.S., Zhang, J., Geng, B., Zhao, J. and Gerns, H. (2006) Apelin protects myocardial injury in- duced by isoproterenol in rats. Regulatory Peptides, 133(1-3), 147-154.

[30] Zhang, Z., Yu, B. and Tao, G.-Z. (2009) Apelin protects against cardiomyocyte apoptosis induced by glucose deprivation. Chiniese Medical Journal, 122(19), 23602365.

[31] Chandrasekaran, B., Dar, O. and McDonagh, T. (2008) The role of apelin in cardiovascular function and heart failure. European Journal of Heart Failure, 10(8), 725-732.

[32] Smith, C.C., Mocanu, M.M. and Bowen, J. (2007) Temporal changes in myocardial salvage kinases during reperfusion following ischemia: Studies involving the cardioprotective adipocytokine apelin. Cardiovascular Drugs and Therapy, 21(6), 409-414.

[33] Cheng, X., Cheng, X.S. and Pang, C.C. (2003) Venous dilator effect of apelin, an endogenous peptide ligand for the orphan APJ receptor, in conscious rats. European Journal of Pharmacology, 470(3), 171-175.

[34] Rastaldo, R., Pagliaro, P., Cappello, S., Penna, C., Mancardi, D., Westerhof, N. and Losano, G. (2005) Nitric oxide and cardiac function. Life Sciences, 81(10), 779793.

[35] Shultz, R., Kelm, M. and Heusch, G. (2004) Nitric oxide in myocardial ischemia/reperfusion injury. Cardiovascular Research, 61(3), 402-413.

[36] Ferrari, R., Ceconi, C., Curello, S., Cargnoni, A., Alfieri, O., Pardini, A., Marzollo, P. and Visioli, O. (1991) Oxygen free radicals and myocardial damage: protective role of thiol-containing agents. The American Journal of Medicine, 91(3), 95S-105S.

[37] Lipasti, J.A., Nevalainen, T.J., Alanen, K.A. and Tolvanen, M.A. (1984) Anaerobic glycolysis and the development of ischaemic contracture in isolated rat heart. Cardiovascular Research, 18(3), 145-148.

[38] Pisarenko, O.I., Lakomkin, V.L., Studneva, I.M., Timoshin, A.A., Kuzmin, A.I., Ruuge, E.K. and Kapelko, V.I. (1994) Allopurinol-enhanced postischemic recovery in the isolated rat heart involves repletion of high-energy phosphates. Biochemical Medicine and Metabolic Biology, 51(1), 16-26.

[39] Rosen, E.D. and Spiegelman, B.M. (2006) Adipocytes as regulatord of energy balance and glucose homeostasis. Nature, 444(7120), 847-853.

[40] Dray, C., Knauf, C., Daviaud, D., Waget, A., Boucher, J., Buleon, M., Cani, P.D., Attane, C. and Guigne, C. (2008) Apelin stimulates glucose utilization in normal and obese insulin-resistant mice. Cell Metabolism, 8(5), 437-445.

[41] Li, J., Hu, X., Selvakumar, P., Russell, R.R., Cushman, S.W., Holman, G.D. and Young, L.H. (2004) Role of the nitric oxide pathway in AMPK-mediated glucose uptake and GLUT4 translocation in heart muscle. American Journal of Physiology-Endocrinology Metabolism, 287(5), E834-E841.

[42] Yue, P., Jin, H., Aillaud, M., Deng, A.C., Azuma, J., Asagami, T. and Kundu, R. K. (2010) Apelin is necessary for the maintenance of insulin sensitivity. American Journal of Physiology-Endocrinology Metabolism, 298(1), E59-E67. 